\title{
A fulminant case of Guillain-Barré syndrome: topographic and fibre size related analysis of demyelinating changes
}

\author{
TAKASHI KANDA, * HIDEAKI HAYASHI, * HITOSHI TANABE, * TADAO TSUBAKI,* \\ MASAYA ODA $\dagger$ \\ From the Departments of Neurology* and Neuropathology, $\uparrow$ Tokyo Metropolitan Neurological Hospital, Fuchu, \\ Tokyo
}

SUMMARY In a necropsy case of early fulminant Guillain-Barré syndrome, demyelinating changes were observed throughout the peripheral nervous system, most conspicuous in the spinal nerve roots. The central/peripheral nervous system transition regions and most proximal zones of the roots escaped damage. In some root areas there were widespread early changes in myelin sheaths in the absence of significant infiltrates of inflammatory cells. In the fibre size analytical study, small myelinated fibres were preferentially involved.

It is now well established that the peripheral nerve damage in Guillain-Barre syndrome (GBS) is not a diffuse process but occurs in the form of discrete foci of inflammation scattered throughout the peripheral nervous system (PNS).$^{12}$ However, the most vulnerable site in this disorder has yet to be completely clarified, and the question as to the spatial distribution of the lesions in the PNS remains unsolved. Although some authors have emphasised abnormalities of spinal roots, ${ }^{34}$ Asbury et al stated that the findings reported previously were only a reflection of the fact that spinal nerve roots had been taken far more frequently than peripheral nerves at necropsy. It was difficult in these previous studies to determine the sites and precise structural changes of demyelination, especially in the initial stage, because the evaluations were based mainly upon the observation of paraffin sections. Hence we used epon-embedded semi-thin sections for evaluating the spatial distribution of demyelination and size distribution of the remaining myelinated fibres in a necropsy case of fulminant, quadriplegic GBS of 8 days' duration.

Address for reprint requests: Dr T Kanda, Department of Neurology, Faculty of Medicine, Tokyo Medical and Dental University, 1-5-45, Yushima, Bunkyo-ku, Tokyo 113, Japan.

Received 21 October 1988 and in revised form 30 December 1988 Accepted 6 February 1989

\section{Case history}

A 47 year old man was admitted to the Tokyo Metropolitan Neurological Hospital on 2 December, 1985, with the complaints of difficulty in breathing, photophobia and descending limb weakness of 36 hours duration. The patient had had an upper respiratory tract infection five days prior to admission which had resolved without treatment. He had been afebrile but had felt hot two days before admission.

His temperature was $36.2^{\circ} \mathrm{C}$, blood pressure $178 / 100 \mathrm{~mm}$ $\mathrm{Hg}$, and pulse rate 130 beats per minute. Electrocardiogram showed a sinus tachycardia. He complained of air hunger, the breathing rate being 24 per minute. He was intubated.

Neurologic examination revealed a fully intact mental status, nasal speech, dysphagia and mild palatal weakness. The fundi were normal but both pupils were dilated to $6 \mathrm{~mm}$ in diameter and non-reactive. The external ocular movements were full but he complained of diplopia in all directions. The limbs showed symmetrical, predominantly proximal weakness and mild ataxia. Sensation was intact and deep tendon reflexes were diminished. Plantar responses were flexor.

Electrolytes, renal and liver functions were normal. The white cell count was $25,900 / \mathrm{mm}^{3}$ with $96 \%$ neutrophils, $19 \%$ band forms, $1 \%$ monocytes and 3\% lymphocytes. A lumbar puncture performed on the second hospital day showed clear colourless cerebrospinal fluid with an opening pressure of 14 $\mathrm{cm}$ of CSF. The protein content was $79 \mathrm{mg} / \mathrm{dl}$. It contained 1 mononuclear cell $/ \mathrm{mm}^{3}$ and $108 \mathrm{mg} / \mathrm{dl}$ of glucose.

Electrophysiological studies were performed on the third hospital day. Motor nerve conduction velocities were normal in the median, ulnar and peroneal nerves, but each $\mathrm{M}$-wave was polyphasic and its amplitude was decreased. Sensory nerve conduction velocities were normal in the median and sural nerves. 
On the second hospital day, the tidal volume was only 300 $\mathrm{ml}$ or less and so he was placed on a respirator. Abduction of both eyes was limited but the Bell's phenomenon was preserved. He could not move his arms against gravity. Deep tendon reflexes had all disappeared except both ankle reflexes.

On the third hospital day, the external ocular movements were severely impaired in all directions and the Bell's phenomenon had disappeared. Deep tendon reflexes were all absent. The upper extremities were totally paralysed and only dorsiflexion of his toes was possible. Plasmapheresis was performed on the third, fourth and fifth hospital days but no signs of immediate improvement were observed.

On the fifth hospital day, all voluntary muscles, including the ocular and palpebral muscles, became completely paralysed. On the sixth hospital day, the pulse rate suddenly decreased from 130 to 30 per minute, and the patient became cyanotic. Resuscitative efforts were unsuccessful; he died on 7 December, 1985.

A necropsy was performed approximately 3 hours after death (No. NHA-124). A relatively fresh small duodenal ulcer (Ul-II) was found and the small intestine was packed with a moderate amount of a tarry material. But this seemed not to have caused the sudden terminal event. Scattered focal pneumonia, a finger tip-sized nodule of a highly differentiated follicular adenocarcinoma in the right lobe of the thyroid, and a small neuroma attached to the S-1 anterior spinal root were found. No particular abnormalities were detected in the brain (weight $1390 \mathrm{~g}$ ) or spinal cord, except for scattered shrunken nerve cells and central chromatolysis of some motor neurons in the brain stem.

\section{Materials and methods}

\section{EXAMINATION OF THE PNS}

For detailed morphological examination and for morphometric analysis, the vagus nerve at the neck, several segments of anterior and posterior roots of the seventh cervical and third lumbar nerve roots, as shown in fig 1 , and the proximal and distal portions of the median, sciatic and

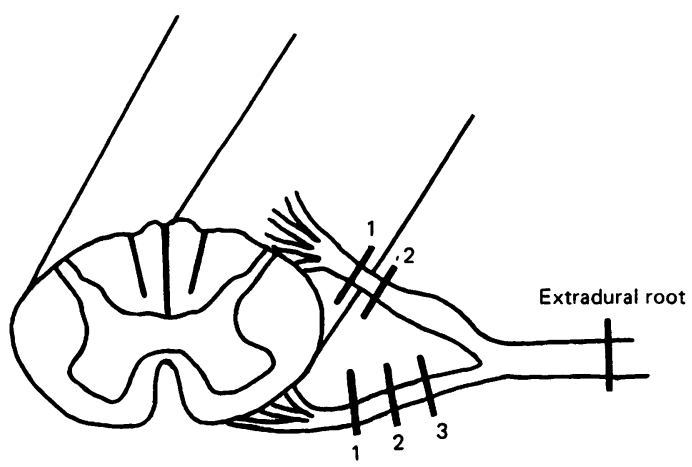

Fig 1 Specimens of the $C 7$ and L3 spinal nerve roots were sampled as shown diagrammatically. They were divided into two (posterior root) or three (anterior root) parts, designated as portions 1, 2 and 3, from the proximal to the distal end. The extradural root of the $C 7$ root was also sampled. sural nerves, were sampled. They were fixed in phosphatebuffered $2.5 \%$ glutaraldehyde, postfixed in $1 \%$ osmic acid and then embedded in Epon.

Toluidine blue-stained semi-thin transverse sections of these materials were used for evaluation of the density and size distribution of the remaining myelinated fibres and used for evaluation of the incidence of demyelinative changes, which were classified into the following two grades: (1) completely demyelinated fibres surrounded by Schwann cells or debris-laden macrophages, and (2) fibres with myelin splitting or disruption, which was interpreted as a relatively early change of demyelination ${ }^{56}$ antedating the former. For quantitative analysis of these changes, about 2,000 myelinated nerve fibres were evaluated in each portion. At least three fascicles in the same transverse plane were randomly chosen to minimise the sampling error.

Three patients who died of cerebrovascular accidents $(56$, 66 and 71 year old men, whose necropsies were performed 3 , 2 and 9 hours, respectively, after death) served as controls for $\mathrm{C} 7$ and $\mathrm{L} 3$ nerve roots. Measurement of the myelinated nerve fibre diameter and statistical analysis were performed using a WT-4400 digitiser (WACOM KK) interfaced to a Nihon Denki PC 9801VM personal computer.

Ultrathin sections were stained with uranyl acetate and lead citrate, and then observed under a JEOL 200CX electron microscope.

Routine neuropathological examination was also performed of formalin-fixed paraffin-embedded samples of additional spinal and cranial nerve roots, peripheral nerve trunks, sympathetic nerves and ganglia, and muscles.

\section{Results}

Myelin-destructive lesions were distributed through out the examined segments of the peripheral nervous system, including the cranial nerves, sympathetic chains and intramuscular nerve twigs. They were patchy or multifocal in both transverse and longitudinal sections. Their severity varied from fascicle to fascicle in the same plane (fig 2). Many phagocytes had proliferated in the oedematous endoneurium along damaged fibres, autophaging myelin debris (figs 2, 3a, and 4a). Many preserved or shadow-like faded axons surrounded by myelin debris were observed in places. On the other hand, the inflammatory cell reaction was rather mild and small lymphocytes were few. Slight lymphocytic infiltrations in the intracardiac ganglia were also seen (fig $4 \mathrm{~b}$, arrows). Figure 5 shows mapping of the lesions from the viewpoint of the demyelinative changes of individual myelinated nerve fibres. These demyelinative changes were never observed in the spinal roots of the controls. Except for the high incidence of fibres with completely degraded myelin sheaths in the vagus nerve (fig $4 a$ ), myelinated fibres in the spinal nerve roots were affected more often than those in main peripheral trunks.

Sensory roots were as severely involved as motor roots, and the paraganglionic, extradural portions of the posterior nerve roots were considered to be the site of maximal attack. 

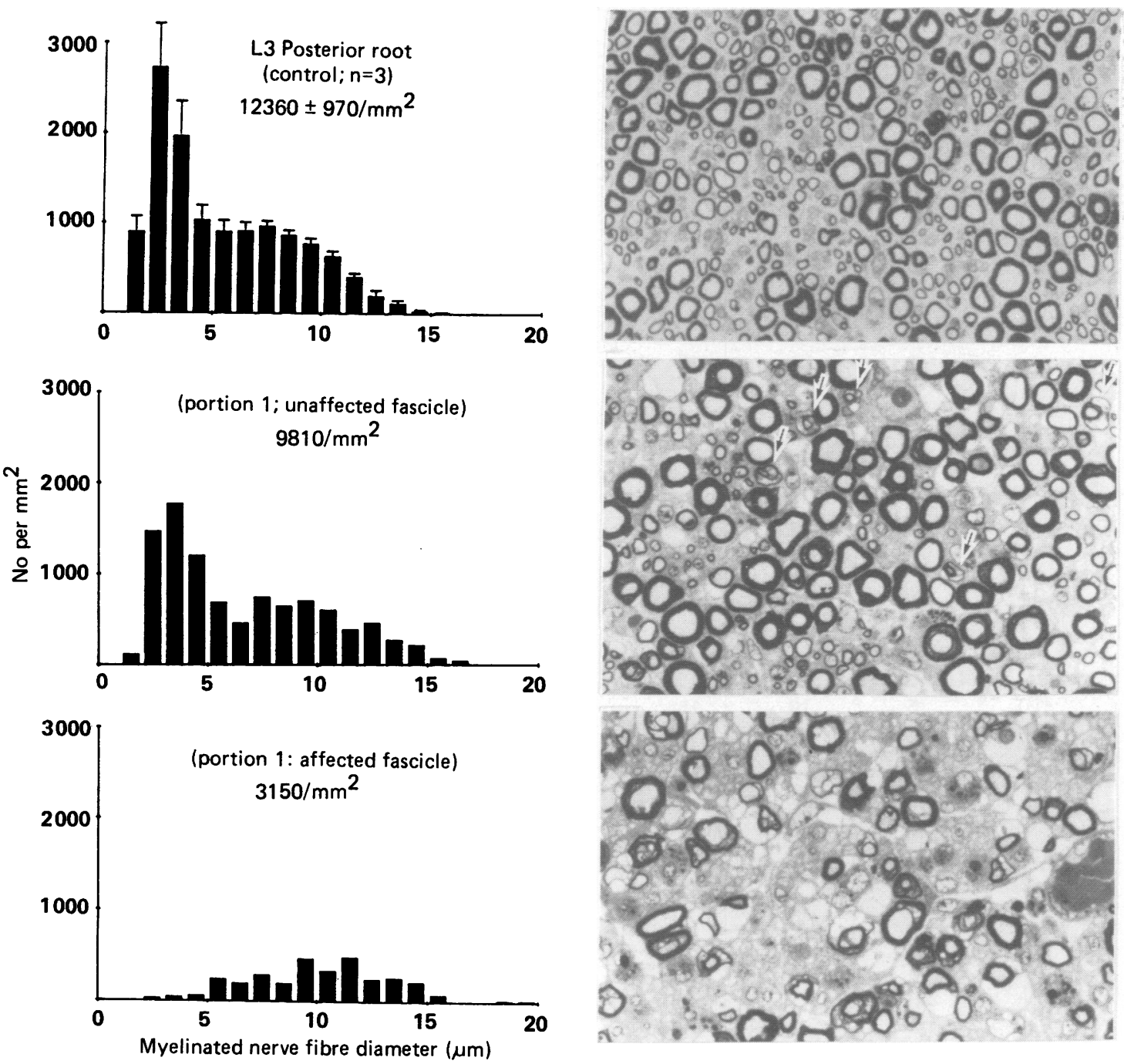

Fig 2 Histologic findings and diameter histograms of the remaining myelinated fibres $/ \mathrm{mm}^{2}$ of the fascicular area of the L3 posterior root, excluding the fibres which had been completely demyelinated. Differential involvement of the adjacent nerve fascicles in the same transverse plane is shown. Upper, controls. The error bars show standard errors of the mean. Middle, portion 1, unaffected fascicle. Although cellular infiltration cannot be observed, there are a small number of fibres with split myelin sheaths (arrows). Slight interstitial oedema may reduce the myelinated nerve fibre density in this area, as compared with that of the controls. Lower, portion 1, affected fascicle. Although fibres with split myelin sheaths were slightly increased in diameter and the diameter histogram of the remaining myelinated fibres can be modified, small fibres with completely degraded myelin sheaths are abundant and preferential involvement of myelin around small axons is highly probable. Semi-thin section. Toluidine blue. $\times 350$.

In the cranial and spinal nerve roots, peripheral myelin located in proximity to the CNS/PNS transitional regions was almost intact and demyelinative changes began abruptly several millimeters distal from the transitional regions (fig 3 ).

Morphometric analysis of the density and fibre size distribution of the remaining myelinated fibres of $\mathrm{C7}$ and L3 spinal nerve roots, excluding the fibres with completely degraded myelin sheaths, revealed the preferential involvement of small myelinated fibres (figs 2 and 3 ).

The electron microscopic study showed no abnor- 

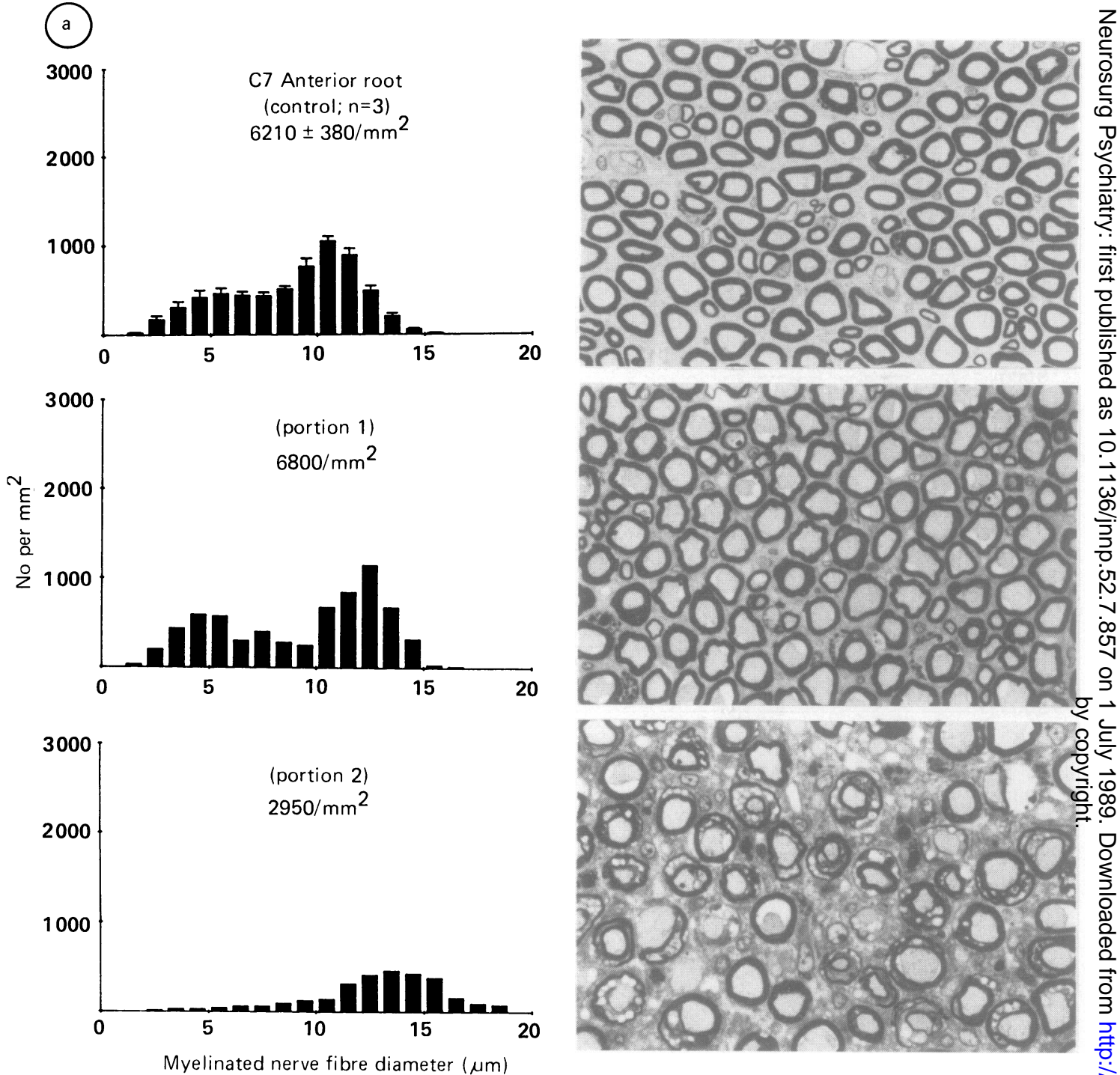

Fig 3 (a) Histologic findings and diameter histograms of the remaining myelinated fibres $/ \mathrm{mm}^{2}$ of the fasicular area of the C7 anterior root. Upper, controls. The error bars show SEM. Middle, portion 1. Although the fibres containing pi granules in the Schwann cell cytoplasm are abundant, the myelinated fibres in this section are not involved and do not show early demyelinative changes. Lower, portion 2. Many of the fibres exhibit myelin disruption: splitting of myelin sheaths. Small fibres with completely degraded myelin sheaths are scattered and there appear to be much more destruction of myelin around small axons than around larger ones. Semi-thin section. Toluidine blue. $\times 350$.

mality in unmyelinated fibres. The most common finding was disruption or degeneration of the myelin sheath with preservation of the axon cylinder. Fibres exhibiting "vesicular disruption" sheaths are abundant (fig 6a). Although some of the denuded axons were shrunken, flattened and displaced laterally by the intruding macrophages, axonal organellae were preserved (fig 6b). Macrophages penetrating through the disrupted basement membranes of myelinated nerve fibres were also observed (fig 6C). These fundamental morphological changes were apparently different from those of primary axonal degen- 


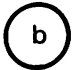

L3 Anterior root
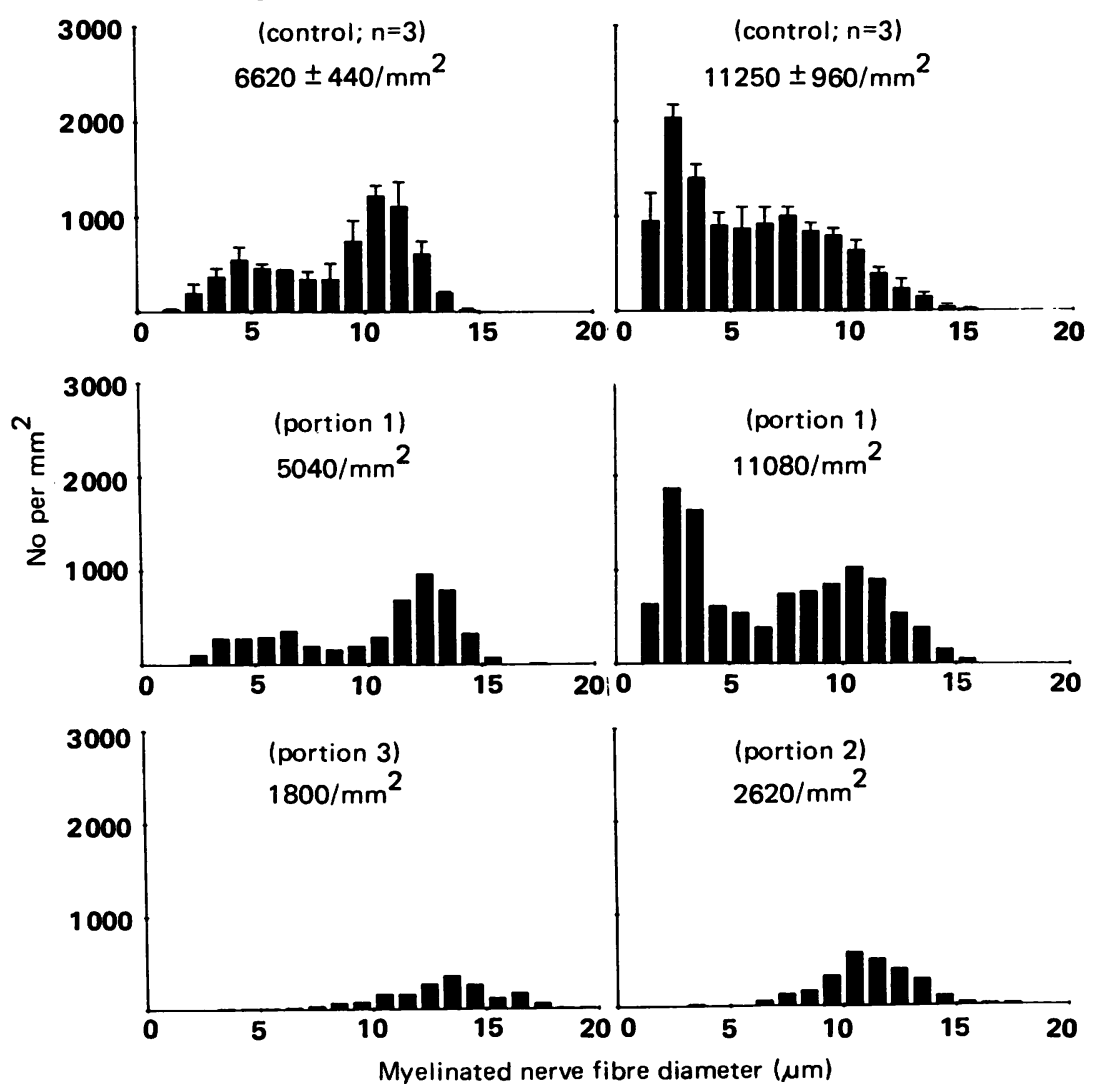

3(b) Preferential involvement of small myelinated nerve fibres is also observed in the $\mathrm{C7}$ posterior root and L3 anterior root. Slight decrease of the myelinated nerve density in the proximalmost, not affected portions as compared with that of the controls may partly be ascribed to the interstitial oedema.

Fig 4 (a) Vagus nerve (neck). Many small fibres are completely demyelinated and intact small fibres are few. Free macrophages autophaging myelin debris, semilunar or triangular in shape, can also be observed (arrows). Fibres exhibiting myelin disruption are relatively rare and demyelinative changes in this portion are considered to be fairly advanced. Semi-thin section. Toluidine blue. $\times 400$. (b) Intracardiac ganglia. Arrows indicate the infiltrating lymphocytes. $H \& E$. $\times 400$ 


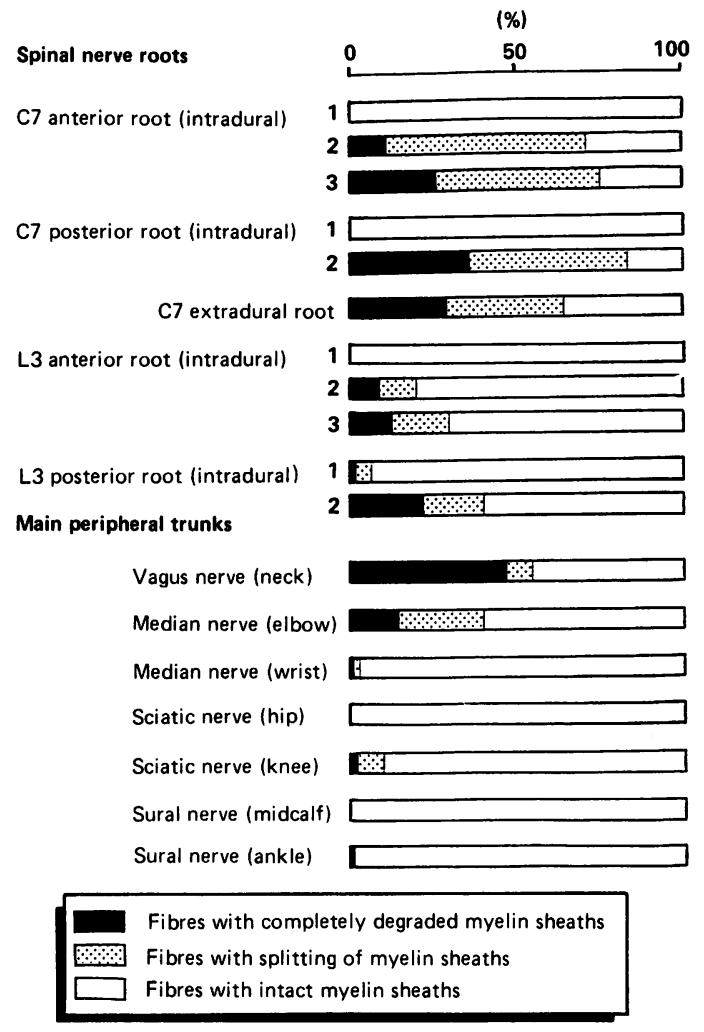

Fig 5 Distribution of the myelinated nerve fibres undergoing demyelinative changes in various portions of the peripheral nervous system. In the spinal nerve roots, areas in proximity to the CNS/PNS transitional regions (portion 1) were almost completely intact, and demyelinative changes appeared distally (portion 2 or 3). As a whole, demyelinated fibres were more prominent in the spinal nerve roots than in the main peripheral trunks except for the vagus nerve. The numbers denote the examined portion, as shown in fig 1 .

eration, although they were at times difficult to be distinguished from "myelin ovoids" on light microscopy.

\section{Discussion}

Although plasmapheresis is said to have been particularly effective for patients who required mechanical ventilation and received this treatment within seven days of onset, ${ }^{10}$ the course of the disease was so aggressive in this patient that he expired before plasmapheresis could be arranged. The marked paralysis and rapid progression to the peak severity in the present case resembled those in the cases of "an acute axonal form" of GBS recently reported by Feasby et al. ${ }^{11}$ However, the findings for transverse semi-thin sections and electron microscopy in the present case (marked myelin breakdown with preservation of axons) revealed that the observed nerve fibre change was essentially acute demyelination as previously reported, ${ }^{72}$ although Wallerian degeneration of various grades was seen. So, this case gave us an opportunity to study the most vulnerable sites of early demyelinative changes of PNS in GBS, because of the unfortunate fulminant course leading to death within 8 days without modification due to the use of corticosteroids or other immunosuppressive drugs.

Using conventional paraffin sections, Asbury et al. ' found that the common denominator in all their 19 cases was perivascular mononuclear inflammatory infiltration and the myelin breakdown was restricted to those regions of nerve trunks infiltrated with inflammatory cells, and concluded that the inflammatory cells might have played a direct role in initiating myelin destruction. However, for quantification and evaluation of precise structural changes of myelinated fibres, observation of paraffin section is less appropriate than that of epon-embedded semithin sections, such as we used. The merits of the latter are that fibre size-related vulnerability can be evaluated and that early demyelinative changes can be easily detected. In this way, we found in our case nerve fibres with myelin splitting or disruption, an early demyelination change, even in regions where the inflammatory cell reaction was not conspicuous. Similar changes in the myelin sheath were also described as the post mortem artefacts observed in the necropsy materialso obtained 20 hours or more after death, ${ }^{13}$ but, in the present case, the necropsy was undertaken as early as 3 hours after death and these changes were never observed in the controls, whose necropsies were performed 2 to 9 hours after death. In addition, the percentage of fibres with splitting of myelin sheaths nearly parallels that of fibres with completely degraded myelin sheaths, as shown in fig 5; these changes in the present case were considered to be genuine, rather than postmortem artefacts.

The reason why the mononuclear infiltrates in our case were less extensive than those in the cases which Asbury et al' described was unknown, but some reports on biopsied materials have been consistent with our findings; Brechenmacher et al. ${ }^{14}$ mentioned that mononuclear cells infiltrating the endoneurium were present in only five out of 57 paraffin-embedded biopsy specimens on light microscopy. Although the lower incidence of mononuclear cell infiltrations in the biopsied materials may be partly ascribed to the smaller size of the specimens, it seems likely that the underlying pathology of GBS is heterogeneous, with some cases involving early perivascular infiltration and being cell-mediated and others involving demyelination without lymphocytic infiltration and 


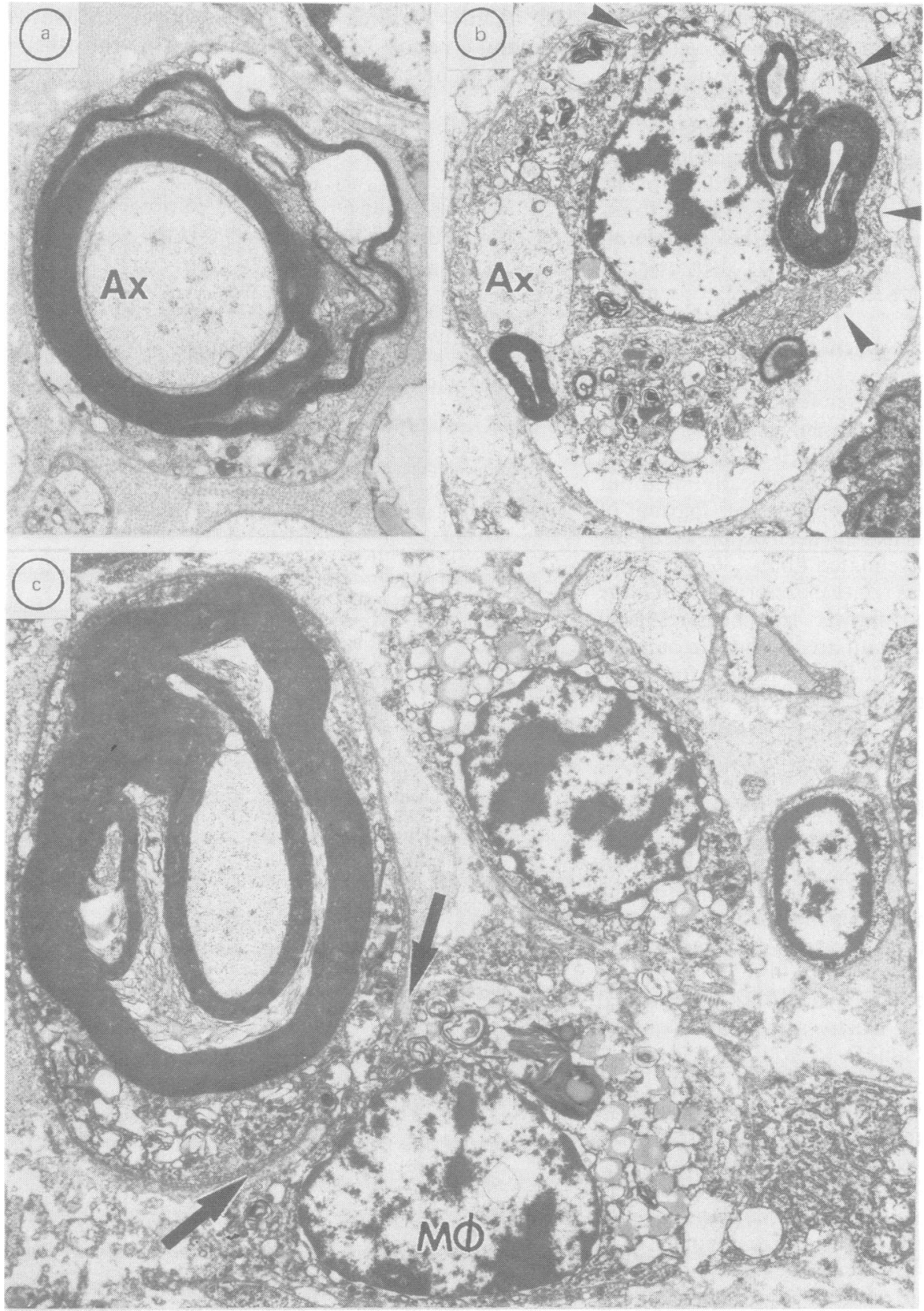

Fig 6 (a) Fibre exhibiting myelin disruption: myelin splitting with vacuolation and vesicular myelin degeneration. The axon is well preserved. $C 7$ extradural root. $\times$ 8,000. (b) Totally demyelinated axon surrounded by macrophages which have invaded beneath the basement membrane. The denuded axon is flattened and displaced laterally but axonal organellae are well preserved. Arrowheads indicate the outline of the intruding macrophage. C7 extradural root. $\times$ 4,800. (c) Macrophage processes penetrate the disrupted basement membrane (arrow). C7 extradural root. $\times$ 5,800 .

being primarily humorally mediated as the present case. The medications without corticosteroids also might be a factor to influence the pathologic picture.

We found also a unique topographic pattern of demyelination in the nerve roots, sparing of the neighbourhood of the CNS/PNS transitional regions (fig 3). The significance of this finding is unknown; however, considering that the early demyelinating changes are thought to be elicited by humoral factors via vessels, the distribution of the vascular supply in 
the CNS/PNS transitional region might play a part. Berthold ${ }^{15}$ pointed out that he found no venule or arteriole in the endoneurial area near the junctional region, where the blood vessels of the PNS compartment did not accompany the nerve bundles but deviated from the rootlet and joined the spinal cord vessels. It is generally accepted that the leakage of the intravascular substances was most prominent in venules when inflammation occurred. ${ }^{16}$ If the demyelinating processes begin at the perivenular sites, relative sparing of the CNS/PNS transitional regions might thus be partly explained.

The preferential involvement of small myelinated fibres in the motor, sensory and autonomic vagus nerves in this study is a matter for consideration. In general, as well as in the present example, GBS is marked by a profound motor deficit with minimal or no objective sensory disturbance clinically. Without overt morphological evidence, relative sparing of cutaneous sensation has usually been explained by the involvement of large myelinated fibres. ${ }^{17}$ Contrary to this, in our case there was relative preservation of large myelinated fibres, as shown by morphological evidence: the motor dominant involvement in our case cannot be explained by either anterior root vulnerability $^{18}$ or preponderance of large fibre involvement.

Other possible hypotheses regarding the motor dominance in GBS are as follows; clinical manifestations of conduction block due to demyelination may be accentuated on motor functions, or motor nerves are selectively involved in the peripheral portions, that is, intramuscular nerve twigs, although with the applied methods and materials in this study we could not answer these questions. For this disorder, further studies including morphometric fibre analysis are needed to clarify whether or not fibre size related vulnerability commonly exists, and studies on the correlation between clinical symptoms and pathological findings should be undertaken.

Although GBS is considered to be a relatively benign disorder, about $5 \%$ of the affected patients die $^{19-22}$ and one of the major causes of sudden death is a severe autonomic disturbance producing cardiovascular disregulations, including fatal arrhythmias. ${ }^{23}$ In the vagus nerve, many of the fibres involved had already been completely demyelinated with or without intact axons, and free macrophages autophaging myelin debris were abundant in the endoneurium (fig 4a). These older appearing lesions in the vagus nerve, in addition to the preferential involvement of myelin sheaths around small axons, may be related to the autonomic derangement (hotness, hypertension and tachycardia) at the beginning of his illness. These changes in the peripheral autonomic nervous system, including the involvement of intracardiac ganglia and sympathetic chains, probably participated in the sudden episode of hypotension and bradycardia at the terminal stage.
We thank Messrs Yoshio Sekiguchi, Mitsuru Takamuku and Kenshiro Kobayashi for their excellent technical assistance.

\section{References}

1 Asbury AK, Arnason BG, Adams RD. The inflammatory lesion in idiopathic polyneuritis. Its role in pathogenesis. Medicine (Baltimore) 1969;48:173-215.

2 Prineas JW. Pathology of the Guillain-Barré syndrome. Ann Neurol 1981;9(suppl):6-19.

3 Casamajor $\mathrm{L}$. Acute ascending paralysis among troops; pathologic findings. Arch Neurol Psychiatry 1919;2:605-20.

4 Haymaker W, Kernohan JW. The Landry-Guillain-Barré syndrome. A clinicopathologic report of fifty fatal cases and a critique of the literature. Medicine (Baltimore) 1949;28: 59-141.

5 Saida K, Sada T, Brown MJ, Silberberg DH, Asbury AK Antiserum-mediated demyelination in vivo. A sequential study using intraneurial injection of experimental allergic neuritis serum. Lab Invest 1978;39:449-62.

6 Hahn AF, Gilbert JJ, Feasby FE. Passive transfer of demyelination by experimental allergic neuritis serum. Acta Neuropathol (Berl) 1980;49:169-76.

7 Carpenter S. An ultrastructural study of an acute fatal case of the Guillain-Barré syndrome. J Neurol Sci 1972;15:125-40.

8 Dal Canto MC, Wisniewski HM, Johnson AB, Brostoff SW, Raine CS: Vesicular disruption of myelin in autoimmune demyelination. J Neurol Sci 1975;24:313-9.

9 Wiesniewski HM, Prineas J, Raine CS. An ultrastructural study of experimental demyelination and remyelination. Part 1: Acute experimental allergic encephalomyelitis in the peripheral nervous system. Lab Invest 1969;21:105-18.

10 The Guillain-Barre Syndrome Study Group. Plasmapheresis and acute Guillain-Barré syndrome. Neurology 1985;35:1096-104.

11 Feasby TE, Gilbert JJ, Brown WF, Bolton CF, Hahn AF, Koopman WF, Zochodne DW. An acute axonal form of Guillain-Barré polyneuropathy. Brain 1986;109:1115-26.

12 Hart MN, Hanks DT, Mackay R. Ultrastructural observations in Guillain-Barré syndrome. Arch Pathol 1972;93:552-5.

13 Hirano A. Some postmortem structural changes in peripheral myelinated fibers. In: Adachi M, Hirano A, Aronson SM, eds. The Pathology of the Myelinated Axon. New York: Igakushoin, 1985:30-48.

14 Brechenmacher C, Vital C, Deminiere C, et al. Guillain-Barré syndrome: Ultrastructural study of peripheral nerve in 65 patients. Clin Neuropathol 1987;6:19-24.

15 Berthold CH, Carlstedt T. Observation on the morphology at the transition between the peripheral and the central nervous system in the cat, II. Acta Physiol Scand suppl 1977;446:23-42.

16 Majno G, Palade GE, Schoefl GI. Studies on inflammation II. The site of action of histamine and serotonin along the vascular tree. J Biophys Biochem Cytol 1961;11:607-25.

17 Asbury AK, Johnson PC. Pathology of Peripheral Nerve. Philadelphia: Saunders, 1978.

18 Kadlubowski M, Hughes RAC, Gregson NA. Spontaneous and experimental neuritis and the distribution of the myelin protein P2 in the nervous system. J Neurochem 1984;42:123-9.

19 Masucci EF, Kurtzke JF. Diagnostic criteria for the GuillainBarré syndrome. An analysis of 50 cases. J Neurol Sci 1971;13:483-501.

20 Samantray SK, Johnson SC, Mathai KV, Pulimood BM. LandryGuillain-Barré-Strohl sydrome. A study of 302 cases. Med J Aust 1977;2:84-91.

21 Moore P, James $\mathrm{O}$. Guillain-Barré syndrome: incidence, management and outcome of major complications. Crit Care Med 1981;9:549-55.

22 Kaur U, Chopra JS, Prabhakar S, Radhakrishnan K, Rana S. Guillain-Barré syndrome. A clinical, electrophysiological and biochemical study. Acta Neurol Scand 1986;73:394-402.

23 Lichtenfield P. Autonomic dysfunction in Guillain-Barré syndrome. Am J Med 1971;50:772-80. 